\title{
Macular perfusion changes assessed with optical coherence tomography angiography after vitrectomy for rhegmatogenous retinal detachment
}

\author{
Hong Wang ${ }^{1,2,3} \cdot$ Xun $X u^{1,2,3} \cdot$ Xiaodong Sun ${ }^{1,2,3} \cdot$ Yingyan $M a^{1,2,3} \cdot$ Tao Sun ${ }^{1,2,3}$ (D)
}

Received: 4 November 2018 / Revised: 31 January 2019 / Accepted: 11 February 2019 / Published online: 22 February 2019

(C) The Author(s) 2019

\begin{abstract}
Purpose To explore macular perfusion changes in patients with rhegmatogenous retinal detachment (RRD) involved the macula following successful surgery and to evaluate the correlation between macular blood flow density and visual outcomes using optical coherence tomography angiography (OCTA).

Methods This retrospective study included 14 eyes (14 patients) with macular-off RRD that underwent a standard three-port 23gauge pars plana vitrectomy (PPV) and intraocular gas tamponade combined with phacoemulsification, aspiration, and intraocular lens implantation. OCTA was used to evaluate the macular perfusion changes throughout postoperative 12 weeks in the superficial capillary plexus (SCP), deep capillary plexus (DCP), and choriocapillary plexus (CCP). The fellow unaffected eyes were used as controls for comparison.

Results A significant increase in the superficial capillary plexus flow density (SCPFD) $(P=0.000)$ was observed over time in RRD eyes with successful PPV, as well as the deep capillary plexus flow density (DCPFD) $(P=0.000)$ and the choriocapillary plexus flow density $(\mathrm{CCPFD})(P=0.000)$. Final best-corrected visual acuity $(\mathrm{BCVA})$ was positively associated with CCPFD $(r=$ $-0.577, P=0.031)$ and non-correlated with SCPFD and DCPFD $(P>0.05)$.

Conclusions Macular perfusion gradually recovered following successful RRD repair by PPV. OCTA provided a non-invasive method to explore the underlying reason for different postoperative visual outcomes in macular-off RRD patients.
\end{abstract}

Keywords Macular perfusion · Optical coherence tomography angiography $\cdot$ Retinal capillary plexus $\cdot$ Choriocapillary plexus · Rhegmatogenous retinal detachment

\section{Introduction}

Rhegmatogenous retinal detachment (RRD), a separation of the sensory retina from the retinal pigment epithelium (RPE), is one of the leading causes for permanent vision loss if untreated. Once the macular fovea is involved in retinal detachment patients, the damage to visual acuity could be severe and irreversible [1].

Tao Sun

drsuntao@yeah.net

1 Department of Ophthalmology, Shanghai General Hospital (Shanghai First People's Hospital), School of Medicine, Shanghai Jiao Tong University, 100 Hai Ning Road, Shanghai 200080, China

2 Shanghai Key Laboratory of Fundus Diseases, Shanghai, China

3 Shanghai Engineering Center for Visual Science and Photomedicine, Shanghai, China
The surgical options include pars plana vitrectomy (PPV) and scleral buckling, which can both achieve amazing final reattachment rates in RRD patients [2]. The retinal and choroidal blood flows reduced following scleral buckling, and the compressional mechanism might be the cause of the reduced blood flow by direct obstruction of the choroidal venous drainage [3, 4]. It has been reported that the ocular microcirculation of RRD patients after PPV could get back to normal 6 months later [5]. Recently, the retinal blood flow on the optic nerve head was proved to be reduced in eyes affected by RRD preoperatively, and it could recover following successful RRD repair by PPV [6]. However, there are only very few studies focusing on the dynamic changes of macular perfusion in eyes with macular-off RRD after operation.

The macular perfusion system, including the superficial capillary plexus (SCP), the deep capillary plexus (DCP), and choriocapillary plexus (CCP), contributes to the metabolic needs of neurons in the visual pathway, which is essential to normal visual function. Optical coherence angiography 
(OCTA) with split-spectrum amplitude decorrelation angiography (SSADA) algorithm, a recently developed clinical tool, provides a non-invasive and quantitative approach for investigating retinal and choroidal microvasculature with high reproducibility [7-9].

The aim of this study is to explore the macular perfusion changes in the PPV-treated eyes with macular-off RRD by comparing the values with those of the fellow unaffected eyes and to evaluate the influence of these changes on the final visual outcome by OCTA.

\section{Material and methods}

This was an observational, cross-sectional, single-center study. A consecutive series of patients were recruited from the inpatients operated at the Department of Ophthalmology, Shanghai General Hospital Affiliated to Shanghai Jiao Tong University, from August 2017 to April 2018. Informed consent was obtained from all the participants included in the study. This study adhered to the tenets of the Declaration of Helsinki and was approved by the Ethics Committee of Shanghai General Hospital affiliated to Shanghai Jiao Tong University.

Only patients with primary recent onset RRD (duration less than 7 days) successfully repaired by PPV were included in this study. The inclusion criteria were (1) unilateral macula-off RRD diagnosed by indirect ophthalmoscopy and B-scan and (2) no postoperative macular complication, including epiretinal membrane, cystoid macular edema, macular hole, and subretinal fluid. The exclusion criteria included the following: (1) history of ocular surgery, (2) other severe visionimpaired eye diseases (e.g., advanced glaucoma or uveitis), (3) best-corrected visual acuity (BCVA) of less than 20/25 in the unaffected fellow eye, and (4) any medical condition that could influence the hemodynamic of the eye such as diabetes, hypertension, arrhythmia, and vascular diseases. All the procedures were performed under retrobulbar anesthesia by the same surgeon (W.H.). Standard three-port 23-gauge PPV was performed using the Alcon Constellation system (Alcon Laboratories, Inc., Fort Worth, TX, USA). After central and peripheral vitreous removal with 23-gauge incision, triamcinolone acetonide was injected to visualize residual posterior hyaloid, which should be removed completely. Complete fluid-air exchange was performed, followed by endophotocoagulation. The sclera ports were sutured with 80 vicryl to maintain normal intraocular pressure (IOP) level. All patients underwent combined phacoemulsification, aspiration, and intraocular lens implantation. And they were asked to maintain a prone posture for 7 days. Retinal reattachment was defined as the complete disappearance of subretinal fluid and flattening of the retinal breaks after gas absorption.
All the 14 participants underwent comprehensive ophthalmic examinations preoperatively and 2, 4, 8, 12 weeks postoperatively, which included slit-lamp biomicroscopy examination, fundus examination, BCVA (the Snellen visual acuity chart), IOP, and OCTA. Values of the unaffected fellow eyes were measured as control.

The technique of OCTA using the SSADA method had been described in detail in previous literatures [8, 10, 11]. The scanning area was captured in $3 \times 3-\mathrm{mm}$ sections, automatically centered on the fovea. The grid was composed of two circles: an inner circle with a diameter of $1 \mathrm{~mm}$ and an outer circle with a diameter of $3 \mathrm{~mm}$. The parafoveal region was defined as the ring between the inner and outer circle (Fig. 2 A1 D1). The macular perfusion parameters, including the superficial capillary plexus flow density (SCPFD), deep capillary plexus flow density (DCPFD), and choriocapillary plexus flow density (CCPFD) in the parafoveal region, were automatically evaluated by RTVue XR Avanti device (Optovue Inc., Fremont, CA, USA) with the AngioVue OCTA software (V2016.2). The ratios of affected eyes and fellow eyes ( $\mathrm{a} / \mathrm{f}$ ratios) were counted to evaluate the dynamic macular perfusion change. The parafoveal retinal thickness (RT) was also analyzed. Thirty minutes before the SSADAOCTA examination, $0.4 \%$ tropicamide/phenylephrine (Mydrin P; Santan Pharmaceutical CO., Ltd., Osaka, Japan) was used to dilate the pupil. The segmentations of all examination were checked before any measurement was performed. With respect to the quality of the images, we took a cutoff value of signal strength index of $>40$ as inclusion criterion for our study.

A paired $t$ test was performed to compare differences between the preoperative and postoperative values and the postoperative values between the affected eyes and the fellow eyes at specific time-points. Repeated one-way ANOVA with post hoc Bonferroni corrections was used to evaluate changes in the BCVA and parafoveal parameters following time. The correlation between final BCVA and the parafoveal parameters was analyzed by Pearson's correlation. The BCVA values were expressed as the logarithm of minimal angle of resolution $(\log M A R)$. Data were presented as mean \pm standard deviation or mean (95\% confidence interval) where applicable. All the tests were performed with SPSS 16.0 for windows (SPSS, Inc., Chicago, IL), and $P$ values $<0.05$ were considered statistically significant.

\section{Results}

\section{Patient demographics and ocular characteristics}

The baseline demographics and ocular characteristics of the enrolled patients are summarized in Table 1. The mean macula-off duration was 4.21 days (median 4 ; range $3 \sim 7$ ). 
Table 1 Patient demographics and ocular characteristics $(n=14)$

Age, mean (range) (years)

$56.0(42 \sim 73)$

Sex (male/female)

$8 / 6$

RRD eye (right/left)

Refractive power, mean (range), diopter

Preoperative BCVA, mean (range), logMAR

RRD eye

Fellow eye

Preoperative IOP, mean (range) $(\mathrm{mmHg})$

RRD eye

Fellow eye

$6 / 8$

$-4.35(-6 \sim-2)$

$1.78(1 \sim 2.3)$

$0.03(0 \sim 0.10)$

$12.36(9 \sim 16)$

$15.86(12 \sim 19)$

$R R D$ rhegmatogenous retinal detachment, $B C V A$ best corrected visual acuity, IOP intraocular pressure

All patients underwent surgery only in the RRD eye throughout the follow-up period. Retinal reattachment was attained at initial operation in all eyes. No significant intraoperative or postoperative complications were observed, such as explosive choroidal hemorrhage, subretinal hemorrhage, endophthalmitis, or anterior proliferative vitreoretinopathy.

\section{Changes in BCVA and parafoveal RT in the eyes with RRD treated by PPV}

A significant improvement was observed in BCVA of the operated eyes with increasing time $(F=91.800, P<0.001)$. The postoperative BCVA (logMAR) was significantly better than preoperative BCVA since 2 weeks after surgery $(t=-$ $8.320, P<0.001$; Table 2). The differences in the BCVA between the operated eyes and the follow eyes decreased from $0.42(0.26$ to 0.58$) \log$ MAR at 2 -week postoperative timepoint to 0.11 ( -0.02 to 0.24$) \log$ MAR at 12 -week postoperative time-point ( $F=35.556, P<0.001$; Table 2$)$.

At each observational postoperative time-point, nonsignificant changes were found between the postoperative parafoveal RTs in the operated eyes and those in the fellow

Table 2 Ocular characteristics in postoperative RRD eyes and unaffected fellow eyes (control) at specific time-point throughout follow-up period

\begin{tabular}{|c|c|c|c|c|c|c|}
\hline & & BCVA (logMAR) & $\mathrm{RT}(\mu \mathrm{m})$ & SCPFD (\%) & DCPFD (\%) & CCPFD (\%) \\
\hline \multirow[t]{6}{*}{2 weeks } & \multirow[t]{2}{*}{ RRD } & 0.46 & 318.43 & 46.06 & 53.67 & 61.15 \\
\hline & & $(0.30 \sim 0.61)$ & (306.42 330.44) & $(43.32 \sim 48.79)$ & $(50.93 \sim 56.41)$ & $(57.98 \sim 64.31)$ \\
\hline & \multirow[t]{2}{*}{ Control } & 0.03 & 311.50 & 54.26 & 60.40 & 67.56 \\
\hline & & $(0.01 \sim 0.06)$ & (301.35 321.65) & $(52.66 \sim 55.86)$ & $(58.22 \sim 62.57)$ & $(64.98 \sim 70.12)$ \\
\hline & \multirow[t]{2}{*}{ Difference } & $-0.42 *$ & -6.93 & $8.21 *$ & $6.73 *$ & $6.41^{*}$ \\
\hline & & $(-0.58 \sim-0.26)$ & $(-18.52 \sim 4.67)$ & $(5.69 \sim 10.72)$ & $(5.24 \sim 8.22)$ & (4.73 8.08) \\
\hline \multirow[t]{6}{*}{4 weeks } & \multirow[t]{2}{*}{ RRD } & 0.27 & 319.57 & 49.18 & 56.50 & 64.62 \\
\hline & & $(0.12 \sim 0.41)$ & $(307.33 \sim 331.81)$ & $(45.92 \sim 52.44)$ & $(54.78 \sim 58.22)$ & $(63.34 \sim 65.89)$ \\
\hline & \multirow[t]{2}{*}{ Control } & 0.03 & 312.43 & 54.87 & 61.28 & 67.69 \\
\hline & & $(0.01 \sim 0.06)$ & $(303.03 \sim 321.82)$ & $(51.35 \sim 58.40)$ & $(59.92 \sim 62.63)$ & $(66.69 \sim 68.81)$ \\
\hline & \multirow[t]{2}{*}{ Difference } & $-0.23^{\#}$ & -7.14 & $5.69^{\#}$ & $4.78^{\#}$ & $3.07^{\#}$ \\
\hline & & $(-0.39 \sim-0.08)$ & $(-17.76 \sim 3.47)$ & $(3.66 \sim 7.72)$ & $(3.30 \sim 6.25)$ & (2.04 4.10) \\
\hline \multirow[t]{6}{*}{8 weeks } & \multirow[t]{2}{*}{ RRD } & 0.17 & 320.50 & 51.11 & 58.51 & 65.94 \\
\hline & & $(0.03 \sim 0.31)$ & $(307.40 \sim 333.60)$ & $(48.81 \sim 53.42)$ & $(56.89 \sim 60.12)$ & $(64.31 \sim 67.57)$ \\
\hline & \multirow[t]{2}{*}{ Control } & 0.03 & 312.00 & 54.77 & 61.14 & 67.19 \\
\hline & & $(0.01 \sim 0.06)$ & $(302.18 \sim 321.82)$ & $(53.35 \sim 56.19)$ & $(59.98 \sim 62.30)$ & $(65.89 \sim 68.50)$ \\
\hline & \multirow[t]{2}{*}{ Difference } & -0.14 & -8.50 & $3.66^{\dagger}$ & $2.63^{\dagger}$ & 1.26 \\
\hline & & $(-0.28 \sim 0.01)$ & $(-19.16 \sim 2.16)$ & $(2.06 \sim 5.26)$ & $(1.53 \sim 3.73)$ & $(-0.02 \sim 2.53)$ \\
\hline \multirow[t]{6}{*}{12 weeks } & \multirow[t]{2}{*}{ RRD } & 0.14 & 319.29 & 53.72 & 59.93 & 66.22 \\
\hline & & $(0.02 \sim 0.26)$ & (306.49 332.08) & $(51.05 \sim 56.40)$ & $(58.51 \sim 61.35)$ & $(64.67 \sim 67.77)$ \\
\hline & \multirow[t]{2}{*}{ Control } & 0.03 & 311.93 & 53.91 & 59.98 & 67.37 \\
\hline & & $(0.01 \sim 0.06)$ & $(302.34 \sim 321.52)$ & $(51.17 \sim 56.65)$ & $(58.56 \sim 61.39)$ & $(66.24 \sim 68.50)$ \\
\hline & \multirow[t]{2}{*}{ Difference } & -0.11 & -7.36 & 0.19 & 0.04 & 1.15 \\
\hline & & $(-0.24 \sim 0.02)$ & $(-18.48 \sim 3.76)$ & $(-0.41 \sim 0.78)$ & $(-0.18 \sim 0.27)$ & $(-0.22 \sim 2.51)$ \\
\hline
\end{tabular}

Data are presented as mean (95\% confidence interval). The differences of the BCVA, RT, SCPFD, DCPFD, and CCPFD were calculated as the values of the control eyes minus those of the RRD eyes

$R R D$ rhegmatogenous retinal detachment, $B C V A$ best-corrected visual acuity, $R T$ retinal thickness, $S C P F D$ superficial capillary plexus flow density, $D C P F D$ deep capillary plexus flow density, $C C P F D$ choriocapillary plexus flow density

$* P<0.05$ (RRD vs. control); ${ }^{*} P<0.05$ (RRD vs. control); ${ }^{\dagger} P<0.05$ (RRD vs. control) 
eyes (all $P>0.05$; Table 2). Parafoveal RT did not change over time in the affected eyes $(F=1.026, P=0.364)$ or in the fellow eyes $(F=0.305, P=0.808)$. The differences in the parafoveal RTs between the operated eyes and the fellow eyes did not show any significant change with postoperative time $(F=0.460, P=0.712)$.

\section{Changes in macular perfusion in the eyes with RRD treated by PPV}

The parafoveal SCPFD and DCPFD were significantly lower in the postoperative eyes than in the fellow eyes until the final observational time-point $(t=-0.671, P=0.514 ; t=-0.423$, $P=0.679$; Table 2). Significant increases with postoperative time were observed in the parafoveal SCPFD and DCPFD of the RRD eyes from $46.06 \pm 4.74 \%$ at 2 weeks postoperatively to $53.72 \pm 4.64 \%$ at 12 weeks postoperatively and from 53.57 $\pm 4.75 \%$ at 2 weeks postoperatively to $59.93 \pm 2.47 \%$ at 12 weeks postoperatively, respectively $(F=23.615$, $P<0.001 ; F=20.210, P<0.001)$. The parafoveal CCPFD in the affected eyes also significantly increased over time $(F=$ 17.437, $P<0.001)$. Compared with the unaffected eyes, the parafoveal CCPFD of the affected eyes was statistically lower at 2 weeks and 4 weeks after operation $(t=-8.265, P<0.001$; $t=-6.425, P<0.001$; Table 2); however, the difference became non-significant since 8 weeks postoperatively $(t=-$ $2.131, P=0.053$; Table 2). There were no significant changes in the parafoveal SCPFD, DCPFD, and CCPFD over time in the fellow eyes $(F=0.268, P=0.740 ; F=1.158, P=0.330$; $F=0.223, P=0.719)$. The absolute differences in the CCPFD, SCPFD, and DCPFD between the RRD eyes and the follow eyes decreased significantly with increasing time postoperatively $(F=30.464, P<0.001 ; F=30.627$, $P<0.001 ; F=50.005, P<0.001)$.

There were significantly differences in the mean a/f ratios of parafoveal SCPFD and DCPFD between 12 weeks postoperatively and other time-points; however, there was no significant difference in the mean $\mathrm{a} / \mathrm{f}$ ratio of parafoveal CCPFD between 12 and 8 weeks postoperatively (Fig. 1). Moreover, the parafoveal CCPFD was significantly greater $(t=3.088, P=0.009)$ in those with better visual outcomes $(B C V A \geq 20 / 25)$ than the others $(B C V A<20 / 25)$ at the last follow-up time-point.

\section{Correlations between final BCVA and parafoveal parameters in the eyes with RRD treated by PPV}

Using univariate analysis, we found that there were no significant correlations between the final BCVA and the preoperative BCVA, preoperative IOP, postoperative IOP, parafoveal RT, parafoveal SCPFD, or parafoveal DCPFD. However, the parafoveal CCPFD was inversely correlated with the final BCVA in the RRD eyes $(r=-0.577, P=0.031$; Table 3$)$.

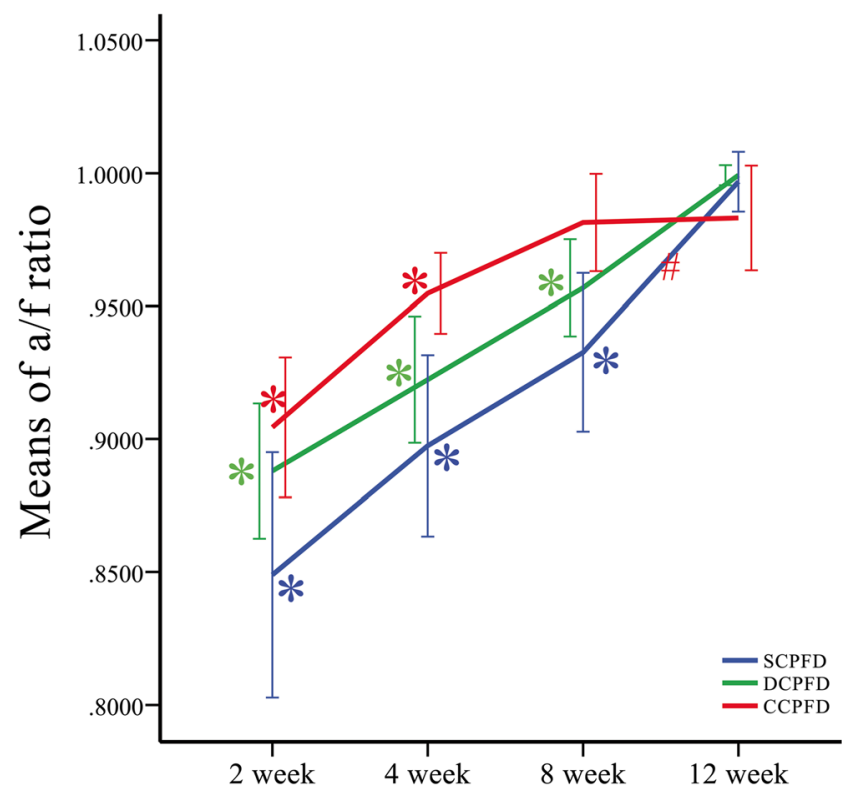

Fig. 1 The time-course of macular perfusion recovery in RRD eyes after successful PPV throughout the follow-up period. The mean a/f (affected eye/fellow eye) ratios of superficial capillary plexus flow density (SCPFD), deep capillary plexus flow density (DCPFD), and choriocapillary plexus flow density (CCPFD) all significantly increased with postoperative time. There were significantly differences in the mean $\mathrm{a} / \mathrm{f}$ ratios of SCPFD and DCPFD between the final follow-up time-point and other time-points ( $* P<0.05$ vs. 12 weeks). Although the mean a/f ratio of CCPFD at 12 weeks post surgery was significantly higher than that at the first two observation time-points $\left({ }^{*} P<0.05\right)$, there was no significant difference in the mean a/f ratio of CCPFD between 8 and 12 weeks postoperatively $\left({ }^{\#} P>0.05\right)$.

Typical images showed that the postoperative structures in the SCP, DCP, and CCP of the patients acquiring final better visual outcome were similar to those in the fellow eyes (Fig. 2 A2 A4 vs. B2 B4). But for the patients with final worse visual acuity, although there were no marked differences in the SCP and DCP structures between the RRD eyes and the unaffected eyes (Fig. 2 C2 C 3 vs. D2 D3), the CCP of RRD eyes were markedly sparse and appeared to be more chaotic comparing with the healthy eyes (Fig. $2 \mathrm{C} 4$ vs. D4). These obvious changes in the CCP structures were also combined with disrupted ellipsoid zone (Fig. 2 C5 vs. D5).

\section{Discussion}

The retinal microcirculation system includes retinal and choroidal capillary networks, which are arranged in different layers. The SCP and the DCP are predominantly located in the ganglion cell layer and the inner nuclear layer, respectively. They provide nourishment to the inner retinal layers and carry metabolic products away. The outer retinal layers are avascular regions which are supplied with nutrients and oxygen by choriocapillaries $[12,13]$. Capillary density can serve as a quantitative measure of 
Table 3 Correlation between final BCVA and clinical parameters at 12 weeks postoperatively in RRD eyes with successful PPV

\begin{tabular}{lll}
\hline & \multicolumn{2}{l}{ Final BCVA (logMAR) } \\
\cline { 2 - 3 } & $r$ & $P$ \\
\hline Preoperative BCVA & 0.239 & 0.410 \\
Preoperative IOP $(\mathrm{mmHg})$ & 0.436 & 0.120 \\
Postoperative IOP $(\mathrm{mmHg})$ & 0.134 & 0.648 \\
Postoperative RT $(\mu \mathrm{m})$ & -0.309 & 0.283 \\
Postoperative SCPFD $(\%)$ & -0.205 & 0.482 \\
Postoperative DCPFD $(\%)$ & -0.236 & 0.416 \\
Postoperative CCPFD $(\%)$ & -0.577 & $0.031^{*}$ \\
\hline
\end{tabular}

Univariate analysis by Pearson correlation

$R R D$ rhegmatogenous retinal detachment, $P P V$ pars plana vitrectomy, $B C V A$ best corrected visual acuity, $I O P$ intraocular pressure, $R T$ retinal thickness, $S C P F D$ superficial capillary plexus flow density, $D C P F D$ deep capillary plexus flow density, $C C P F D$ choriocapillary plexus flow density

$* P<0.05$

detecting flow change caused by physiological or pathological reasons $[14,15]$.

Previous reports using blood flow detection techniques, including scanning laser Doppler flowmetry [5], color Doppler ultrasonography [16], and laser speckle flowgraphy [6], reported reduced retinal blood flow in retinal detachment patients. Nowadays, OCTA has been used to measure capillary density in healthy eyes and in several diseased states because it allows for acquisition of high-resolution depth-resolved images of the chorioretinal vascular layers in a rapid, non-invasive manner without dye injection [8, 17]. Moreover, OCTA is sensitive in detecting the subclinical microvascular changes, including parafoveal capillary density loss, in some retinal diseases such as diabetic retinopathy and radiation maculopathy $[18,19]$. The tortuous and vertically ascended retinal vessels in macular-off RRD eyes make it difficult to estimate the preoperative macular perfusion. Previous studies had proved that macular microcirculation disturbance was present in RRD patients even without macular involvement [3]. In the present study, we applied OCTA to analyze the parafoveal flow density per layer and confirmed that macular perfusion decreased in the macular-off RRD eyes even after successful PPV in comparison to the healthy fellow eyes. The presumption of macular microcirculation seriously impaired in macular-off RRD patients was proved to be reasonable.

In order to know the macular perfusion change in macularoff RRD eyes over time after PPV, we evaluated the perfusion parameters at different time-points. Our results discovered that the parafoveal flow density of the SCP and DCP in the RRD eyes increased significantly over time during the whole observation period, and the differences between the RRD eyes and the follow eyes decreased with increasing time after surgery. Recently, the deep foveal avascular zone (FAZ) area of macular-off RRD eyes was proved to become larger than the unaffected fellow eyes 2 months after surgery [20]. It was consistent with our results that the retinal perfusion in the RRD eyes was still not up to the normal level at postoperative 8 weeks time-point, which might lead to macular hypoxia and FAZ enlargement. In addition, we also noticed the different rehabilitation curves of macular microcirculation in retinal layer and choroidal layer. The rehabilitation curve of CCPFD achieved plateau period ahead of the rehabilitation curve of SCPFD and DCPFD. These findings suggested that the postoperative macular perfusion gradually recovered along with the attached and flattened retina, and there existed a degree of difference in the recovery speed between the retinal circulation and the choroidal circulation. In previous reports, the impairment of microcirculation in RRD patients had been confirmed and the normalization of microcirculation needed 6 months or even more time to be achieved after successful surgery $[5,21,22]$. The discrepancy of recovery speed between our findings and the previous studies might be due to the characteristics of the eyes studied, surgical procedures, measurement methods, and measured areas.

Interestingly, the postoperative CCPFD rehabilitation curve was similar to the postoperative visual acuity rehabilitation curve presented in our study. It has been demonstrated that the parafoveal retinal vascular could response to visual stimulation, suggesting the correlation between the macular perfusion and light activation and adaptation of photoreceptors [7]. Recently, the 3-month postoperative SCP vessel density and DCP vessel density were proved not to be correlated with 3-month postoperative VA in macular-off RRD patients [23], which was consistent with our results. Instead, the 12-week postoperative BCVA was detected to be positively correlated with the CCPFD in our study. Furthermore, we found that the patients with worse visual outcome appeared to have abnormal ellipsoid zone structure associated with the relatively descending choroidal blood flow. It had been reported that the increase in BCVA accompanied with the improvement in outer retinal morphology was significantly correlated with the macular choroidal circulation elevation in eyes with acute zonal occult outer retinopathy, multiple evanescent white dot syndrome, and chorioretinopathy associated with ocular blunt trauma [24-26]. Furthermore, the choriocapillaris layer beneath the disrupted ellipsoid zone of the photoreceptor had greater areas of flow void than the area beneath an intact ellipsoid zone in ischemic maculopathy [27]. Our results were consistent with the previous studies which suggested that the recovery of impaired choroidal circulation was important for the improvements in visual function and outer retinal morphology in retinal diseases. 

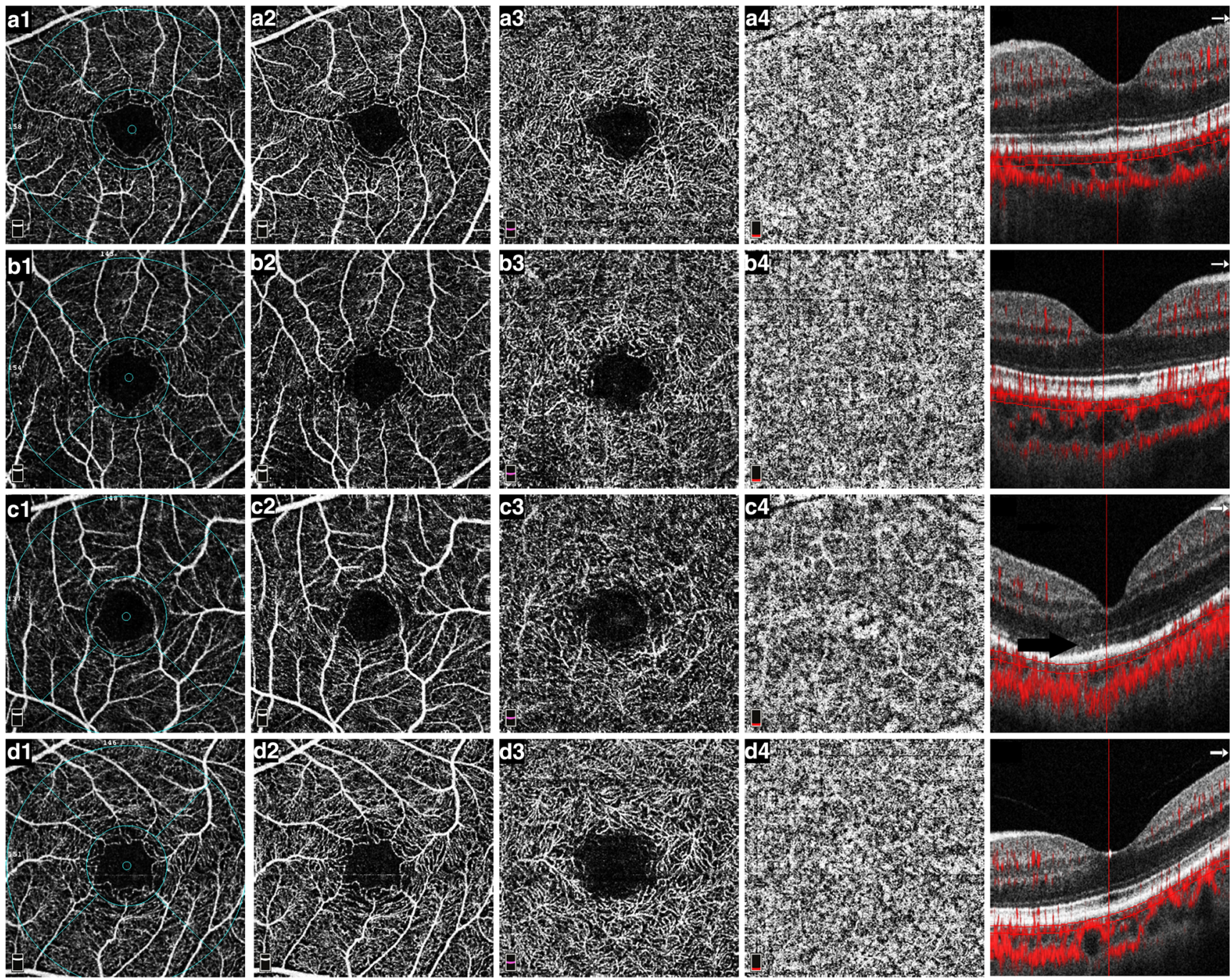

Fig. 2 Representative optical coherence tomography angiography (OCTA) images of the macular perfusion in the RRD eyes and fellow eyes at the last assessment time-point. Representative OCTA images of two male patients (patient 1: A, B; patient 2: C, D) with successful macular-off rhegmatogenous retinal detachment (RRD) repair. The parafoveal region was defined as the ring between the inner and outer circle (A1 D1). The other images showed the superficial capillary plexus (SCP: A2 D2), deep capillary plexus (DCP: A3 D3), choriocapillary plexus (CCP: A4 D4), and macular ultrastructure (A5 D5) of the affected eye $(\mathrm{A}, \mathrm{C})$ and the unaffected fellow eye $(\mathrm{B}, \mathrm{D})$ at postoperative 12 weeks, respectively. Final $\log$ MAR best-corrected visual acuity (BCVA) of the RRD eye was 0.00 for patient 1 and 0.82 for patient 2. BCVA of the fellow eyes were both 0.00 . CCP in the RRD eye of patient $2(\mathrm{C} 4)$ appeared to be more sparse and chaotic comparing to the normal compact structure (D4) in the fellow eye. Longitudinal scans showed disruption of ellipsoid zone (C5, rightwards arrow) in the RRD eye and integrated ellipsoid zone in the fellow eye (D5) of patient 2. Automatic analyses for images (A4 D4) showed choriocapillary plexus flow density (CCPFD) of $66.94 \%, 68.25 \%, 62.07 \%$, and $69.21 \%$, respectively. Patient 2 with worse visual acuity appeared to present abnormal CCP and disrupted ellipsoid zone accompanied by decreased CCPFD
Our study had several limitations. First, a selection bias may have been introduced for its retrospective design and one single hospital's data. Second, we did not evaluate the height and volume of the macular detachment, which might affect the postoperative macular perfusion results. And third, the sample size was relatively small and the postoperative follow-up period was relatively short. Further prospective randomized study with a larger number of patients and a longer observation period should be performed to confirm these results.
In conclusion, the quantitative OCTA analyses confirmed that the postoperative parafoveal flow density in the superficial, deep, and choriocapillaris layers decreased in the macular-off RRD eyes compared with the unaffected fellow eyes. Macular perfusion gradually improved over time following successful RRD repair by PPV. Evaluation of choroidal microcirculation using noninvasive OCTA could be a potential indicator for explaining different postoperative visual outcome in macular-off RRD patients. 
Funding This study was funded by the National Natural Science Foundation for Young Scholars of China (grant number 81200701). The funding organization had no role in the design or conduct of this research.

\section{Compliance with ethical standards}

Conflict of interest The authors declare that they have no conflict of interest.

Ethical approval All procedures performed in this study involving human participants were in accordance with the ethical standards of the Ethics Committee of Shanghai General Hospital affiliated to Shanghai Jiao Tong University and with the 1964 Helsinki declaration and its later amendments or comparable ethical standards.

Informed consent Informed consent was obtained from all individual participants included in the study.

Open Access This article is distributed under the terms of the Creative Commons Attribution 4.0 International License (http:// creativecommons.org/licenses/by/4.0/), which permits unrestricted use, distribution, and reproduction in any medium, provided you give appropriate credit to the original author(s) and the source, provide a link to the Creative Commons license, and indicate if changes were made.

Publisher's note Springer Nature remains neutral with regard to jurisdictional claims in published maps and institutional affiliations.

\section{References}

1. D'Amico DJ (2008) Clinical practice. Primary retinal detachment. N Engl J Med 359:2346-2354. https://doi.org/10.1056/ NEJMcp0804591

2. Sun Q, Sun T, Xu Y, Yang XL, Xu X, Wang BS, Nishimura T, Heimann H (2012) Primary vitrectomy versus scleral buckling for the treatment of rhegmatogenous retinal detachment: a metaanalysis of randomized controlled clinical trials. Curr Eye Res 37: 492-499. https://doi.org/10.3109/02713683.2012.663854

3. Eshita T, Shinoda K, Kimura I, Kitamura S, Ishida S, Inoue M, Mashima Y, Katsura H, Oguchi Y (2004) Retinal blood flow in the macular area before and after scleral buckling procedures for rhegmatogenous retinal detachment without macular involvement. Jpn J Ophthalmol 48:358-363. https://doi.org/10.1007/s10384004-0096-5

4. Sugawara R, Nagaoka T, Kitaya N, Fujio N, Takahashi J, Takahashi A, Yokota H, Yoshida A (2006) Choroidal blood flow in the foveal region in eyes with rhegmatogenous retinal detachment and scleral buckling procedures. Br J Ophthalmol 90:1363-1365. https://doi. org/10.1136/bjo.2006.097485

5. Sato EA, Shinoda K, Kimura I, Ohtake Y, Inoue M (2007) Microcirculation in eyes after rhegmatogenous retinal detachment surgery. Curr Eye Res 32:773-779. https://doi.org/10.1080/ 02713680701531108

6. Iwase T, Kobayashi M, Yamamoto K, Yanagida K, Ra E, Terasaki H (2016) Changes in blood flow on optic nerve head after vitrectomy for rhegmatogenous retinal detachment. Invest Ophthalmol Vis Sci 57:6223-6233. https://doi.org/10.1167/iovs.16-20577

7. Wei E, Jia Y, Tan O, Potsaid B, Liu JJ, Choi W, Fujimoto JG, Huang D (2013) Parafoveal retinal vascular response to pattern visual stimulation assessed with OCT angiography. PLoS One 8:e81343. https://doi.org/10.1371/journal.pone.0081343

8. Yu J, Jiang C, Wang X, Zhu L, Gu R, Xu H, Jia Y, Huang D, Sun X (2015) Macular perfusion in healthy Chinese: an optical coherence tomography angiogram study. Invest Ophthalmol Vis Sci 56:3212 3217. https://doi.org/10.1167/iovs.14-16270

9. Sugahara M, Miyata M, Ishihara K, Gotoh N, Morooka S, Ogino K, Hasegawa T, Hirashima T, Yoshikawa M, Hata M, Muraoka Y, Ooto S, Yamashiro K, Yoshimura N (2017) Optical coherence tomography angiography to estimate retinal blood flow in eyes with retinitis pigmentosa. Sci Rep 7:46396. https://doi.org/10.1038/ srep46396

10. Spaide RF, Klancnik JJ, Cooney MJ (2015) Retinal vascular layers imaged by fluorescein angiography and optical coherence tomography angiography. JAMA OPHTHALMOL 133:45-50. https:// doi.org/10.1001/jamaophthalmol.2014.3616

11. Agemy SA, Scripsema NK, Shah CM, Chui T, Garcia PM, Lee JG, Gentile RC, Hsiao YS, Zhou Q, Ko T, Rosen RB (2015) Retinal vascular perfusion density mapping using optical coherence tomography angiography in normal and diabetic retinopathy patients. Retina 35:2353-2363. https://doi.org/10.1097/IAE. 0000000000000862

12. Snodderly DM, Weinhaus RS, Choi JC (1992) Neural-vascular relationships in central retina of macaque monkeys (Macaca fascicularis). J Neurosci 12:1169-1193

13. Kur J, Newman EA, Chan-Ling T (2012) Cellular and physiological mechanisms underlying blood flow regulation in the retina and choroid in health and disease. Prog Retin Eye Res 31:377-406. https://doi.org/10.1016/j.preteyeres.2012.04.004

14. Chan G, Balaratnasingam C, Yu PK, Morgan WH, McAllister IL, Cringle SJ, Yu DY (2012) Quantitative morphometry of perifoveal capillary networks in the human retina. Invest Ophthalmol Vis Sci 53:5502-5514. https://doi.org/10.1167/iovs.12-10265

15. Chan G, Balaratnasingam C, Yu PK, Morgan WH, McAllister IL, Cringle SJ, Yu DY (2013) Quantitative changes in perifoveal capillary networks in patients with vascular comorbidities. Invest Ophthalmol Vis Sci 54:5175-5185. https://doi.org/10.1167/iovs. 13-11945

16. Roldan-Pallares M, Musa AS, Hernandez-Montero J, Bravo-Llatas SC, Fernandez-Durango R (2013) Retinal detachment and proliferative vitreoretinopathy: central retinal artery blood velocities, intraocular pressure, and endothelin 1. Retina 33:1528-1539. https:// doi.org/10.1097/IAE.0b013e318285cbef

17. Huang D, Jia Y, Gao SS, Lumbroso B, Rispoli M (2016) Optical coherence tomography angiography using the optovue device. Dev Ophthalmol 56:6-12. https://doi.org/10.1159/000442770

18. Tam J, Dhamdhere KP, Tiruveedhula P, Manzanera S, Barez S, Bearse MJ, Adams AJ, Roorda A (2011) Disruption of the retinal parafoveal capillary network in type 2 diabetes before the onset of diabetic retinopathy. Invest Ophthalmol Vis Sci 52:9257-9266. https://doi.org/10.1167/iovs.11-8481

19. Say EA, Samara WA, Khoo CT, Magrath GN, Sharma P, Ferenczy S, Shields CL (2016) Parafoveal capillary density after plaque radiotherapy for choroidal melanoma: analysis of eyes without radiation maculopathy. Retina 36:1670-1678. https://doi.org/10.1097/ IAE.0000000000001085

20. Woo JM, Yoon YS, Woo JE, Min JK (2018) Foveal avascular zone area changes analyzed using OCT angiography after successful rhegmatogenous retinal detachment repair. Curr Eye Res 43:674678. https://doi.org/10.1080/02713683.2018.1437922

21. Ito Y, Sasoh M, Ido M, Osawa S, Wakitani Y, Uji Y (2005) Effects of scleral buckling without encircling procedures on retrobulbar hemodynamics as measured by color Doppler imaging. Arch Ophthalmol 123:950-953. https://doi.org/10. 1001/archopht.123.7.950 
22. Yokota H, Mori F, Nagaoka T, Sugawara R, Yoshida A (2005) Pulsatile ocular blood flow: changes associated with scleral buckling procedures. Jpn J Ophthalmol 49:162-165. https://doi.org/10. 1007/s10384-004-0161-0

23. Yui N, Kunikata H, Aizawa N, Nakazawa T (2018) Optical coherence tomography angiography assessment of the macular capillary plexus after surgery for macula-off rhegmatogenous retinal detachment. Graefes Arch Clin Exp Ophthalmol. https://doi.org/10.1007/ s00417-018-4133-3

24. Saito M, Saito W, Hashimoto Y, Yoshizawa C, Shinmei Y, Noda K, Ishida S (2014) Correlation between decreased choroidal blood flow velocity and the pathogenesis of acute zonal occult outer retinopathy. Clin Exp Ophthalmol 42:139-150. https://doi.org/10. 1111/ceo.12143

25. Hashimoto Y, Saito W, Saito M, Hirooka K, Mori S, Noda K, Ishida S (2015) Decreased choroidal blood flow velocity in the pathogenesis of multiple evanescent white dot syndrome. Graefes Arch Clin Exp Ophthalmol 253:1457-1464. https://doi.org/10. 1007/s00417-014-2831-Z

26. Ishikawa Y, Hashimoto Y, Saito W, Ando R, Ishida S (2017) Blood flow velocity and thickness of the choroid in a patient with chorioretinopathy associated with ocular blunt trauma. BMC Ophthalmol 17:86. https://doi.org/10.1186/s12886-017-0480-9

27. Dodo Y, Suzuma K, Ishihara K, Yoshitake S, Fujimoto M, Yoshitake T, Miwa Y, Murakami T (2017) Clinical relevance of reduced decorrelation signals in the diabetic inner choroid on optical coherence tomography angiography. Sci Rep 7:5227. https:// doi.org/10.1038/s41598-017-05663-9 